\title{
CrystEngComm
}

Cite this: CrystEngComm, 2014, 16, 690

Received 24th July 2013, Accepted 21st October 2013

DOI: $10.1039 / c 3 c e 41467 a$

www.rsc.org/crystengcomm

\section{An investigation into the effect of mixing on the secondary nucleation of sodium chlorate in a stirred tank and an oscillatory baffled crystallizer $\dagger$}

\author{
Craig J. Callahan and Xiong-Wei $\mathrm{Ni}^{*}$
}

\begin{abstract}
This paper reports the outcome of the examination of the effect of mixing intensity on the secondary nucleation mechanism of seeded crystallization of sodium chlorate in various configurations of stirred tank crystallizer (STC) and oscillatory baffled crystallizer (OBC). The results show that for the STC, an un-scraped system always yielded crystals of the same enantiomorphism as the seed crystal. The introduction of scraping to the STC resulted in product crystals of the opposite enantiomorphism to the seed being formed, however changing the rate of stirring (RPM) had no influence over the percentage similarity to the seed crystal. For the OBC, scraping always gave a product crystal crop with less than $100 \%$ similarity to the seed. Similarly, this was unaffected by altering the frequency of oscillation. Removing the scraping effect brought an increase of the similarity to the seed but the product crystals never reached $100 \%$ similarity to the seed enantiomorphism. For both scraped and un-scraped OBCs, reduction of the oscillation amplitude did increase the similarity of the products to the seed and $100 \%$ similarity to the seed was repeatedly achieved at the lowest operating amplitude for the un-scraped OBC. These findings provide further insight into the nucleation mechanism in the $\mathrm{OBC}$ and suggest that an alternative mechanism to that in the STC is observed.
\end{abstract}

\section{Introduction}

Over a hundred years ago, Young described how agitation would cause the onset of nucleation in a quiescent, supersaturated solution in which no crystallization would occur spontaneously. ${ }^{1}$ This has since been studied in more detail and it is now recognized that mixing influences the metastable zone width (MSZW), ${ }^{2}$ an important scientific parameter of solution crystallization. Some early works by Mullin and Raven showed that for the batch cooling crystallization of some aqueous salts, increasing the agitation rate raised the nucleation temperature; further increasing the stirrer speed reduced the nucleation temperature slightly and further intensifying the agitation rate resulted in enhanced nucleation with higher nucleation temperature once again. The suggested mechanism for this phenomenon was a highly complex combination of enhanced mass transfer due to reduced diffusion distance competing against disruption of pre-nuclei clusters due to fluid shear. ${ }^{3,4}$

EPSRC Centre for Innovative Manufacturing in Continuous Manufacturing and Crystallisation, Centre for Oscillatory Baffled Reactor Applications (COBRA), School of Engineering and Physical Science, Heriot-Watt University, Edinburgh, EH14 4AS, UK. E-mail: x.ni@hw.ac.uk; Tel: +44 (o) 1314513781

$\dagger$ Electronic supplementary information (ESI) available. See DOI: 10.1039/ c3ce41467a
Nývlt et al. investigated the relationship between stirrer speed and MSZW in order to probe the primary nucleation kinetics of aqueous solutions. They found that the higher the stirrer speed, the higher the nucleation rate constant, but the nucleation order was only dependent on the number of molecules required to form a critical nucleus, i.e. the nucleation order was independent of agitation rate. ${ }^{5,6}$

The relationship between nucleation and agitation rates investigated by Mullin and Nývlt were recently observed by Liang et al. in the study of L-glutamic acid crystallization. They hypothesised that reduction of boundary layer thickness was observed at higher agitation rates, which caused the change in nucleation rates, ${ }^{7}$ although this parameter is difficult to observe and measure.

The mixing effect applies not only to primary nucleation, but also to secondary nucleation. ${ }^{8}$ Melia and Moffitt presented data on the secondary nucleation of potassium chloride from aqueous solution and suggested two possible mechanisms for the secondary nucleation process. ${ }^{9}$ The first possibility was that in the vicinity of the seed crystal, weakly attracted solute molecules were somewhat ordered in the seed crystal boundary layer without actually being incorporated into the crystal structure. These clusters remained in the boundary layer and, with increasing fluid shear, the boundary layer could be washed into the bulk solution and replaced with fresh solution. These clusters then developed into nuclei in the bulk solution away 
from the seed crystal. This type of nucleation was reported earlier by Powers in the study of sucrose crystallization. ${ }^{10}$

The second mechanism was that the solute molecules were incorporated into the seed crystal structure as dendrites. Through the action of fluid shear, or by crystallizer-crystal collisions, these dendrites could be ripped from the seed crystal and carried into the bulk solution to further grow. Melia and Moffitt supported this type of mechanism for the crystallization of ammonium chloride. ${ }^{11}$ It would be reasonable to assume that these mechanisms are dependent on agitation rate.

Min and Goldburg investigated the influence of fluid shear on the formation of 2,6-lutidine droplets in water. In their experiments, the droplets appeared as temperature increased. Increasing the fluid shear resulted in a reduction of the embryo-type clusters and reduced the effective droplet nucleation rate. ${ }^{12}$ This outcome is particularly relevant to the implementation of a two-step nucleation model where pre-nucleation clusters may exist as a dense fluid phase, ${ }^{13}$ and relevant to what may occur in the boundary layer of a seed crystal when fluid dynamics of a crystallizer are altered.

The combined actions of collision breeding and the boundary layer models were discussed by Buhse et al. who concluded that secondary nucleation by either model was dependent on the crystallizer's hydrodynamics. ${ }^{14}$ Chen et al. found that the thickness of sodium chlorate boundary layer was dependent on the fluid dynamic conditions. ${ }^{15}$ It seems likely that the boundary layer bred nucleation process was competing with the collision bred one; the ratio of the two would depend on not only crystallizer hydrodynamics but also its fluid dynamics.

In general it has been shown that by altering the crystallizer's fluid dynamics, it becomes possible to manipulate many important factors critical to nucleation. Increasing fluid agitation intensity and shear rates tends to reduce boundary layer thickness and would have measurable effects on what is happening in the vicinity of a seed crystal. Fluid shear may also influence any pre-nucleation clusters that may exist in the system ${ }^{16}$ and allow some degree of impact over nucleation rates.

It has previously been stated that "crystallization may be the most difficult operation to scale-up - successfully" and highlighted the importance of mixing at all scales for control of nucleation and subsequent growth. ${ }^{17} \mathrm{~A}$ drive to minimize differences in fluid dynamics at different scales of operation has led to the use of continuous crystallization by means of plug flow crystallizers such as the continuous oscillatory baffled crystallizer (COBC). ${ }^{18,19}$ Due to the uniform mixing, plug flow characteristics, ${ }^{20}$ enhanced mass ${ }^{21}$ and heat transfer rates, ${ }^{22}$ together with the ease and readiness of controlling temperature profiles along the length of the crystallizer, this provides a constant fluid mechanical condition for the nucleation and subsequent growth of crystals. This has led to uniform crystal sizes with significantly enhanced filtration rates. $^{23}$ The scale up of a lab scale COBC of typically 10-15 mm diameter to a pilot/full scale COBC of 40-100 mm diameter is a linear process based on the maintenance of the vessel geometry such as the baffle spacing and the orifice to tube cross sectional area ratio regardless of vessel length. ${ }^{24}$ The ability to scale up in such a way means that there is little variation in fluid mechanical conditions between lab pilot and full modes of operation. This results in little variation of metastable zone widths and facilitates a direct and smooth scale up operation. In addition, analytical tools and monitoring techniques can be used at all scales without modification.

Typically, in order to successfully implement a continuous crystallizer, the process will be performed on the batch scale in order to confirm the kinetics (MSZW, nucleation mechanisms and growth mechanisms) and examine the effects of operating parameters on crystal specification. From those, the residence time for a COBC can be determined, which is roughly equal to the differences between the starting and the nucleation temperature and between the nucleation temperature and the end temperatures divided by suitable desirable cooling rates. It is therefore the scope of this work to understand how the fluid dynamics utilised in batch operation can assist the decisions to be made in continuous processing, and gain some insights as to how a crystallizing species may behave in a continuous crystallizer.

In recent studies of crystallization of active pharmaceutical ingredients (APIs) in the OBC, some interesting results were consistently observed. For example, higher nucleation temperatures and narrower MSZW was observed in an OBC than in a stirred tank crystallizer (STC), even though processes parameters, such as solution concentration, cooling rates and mixing intensity, i.e. power dissipated in the solution by the mixing device, remained constant. ${ }^{25}$ Rapid growth of crystals in an OBC without the entrainment of impurities was possible ${ }^{26}$ as was seeding being unnecessary in an $\mathrm{OBC}$ to obtain a certain crystal specification while it was essential in an STC for the same operation. ${ }^{27}$ This current work aims to address the latter finding by utilizing a crystal compound whose enantiomorphism or morphology in the solid state could be related to the nucleation mechanism. Sodium chlorate is such a non-chiral substance that on crystallization produces crystalline solids possessing either a left (levorotatory) or right handed (dextrorotatory) enantiomorphism. ${ }^{28}$ As such, the crystalline solid will display optical activity, a property that has been exploited in previous studies to determine the handedness of the crystals based on the direction of rotation of plane polarized light, ${ }^{29,30}$ and in turn elucidate the origin of the product crystals. ${ }^{31,32}$

Sodium chlorate was previously utilised in two relevant investigations into nucleation mechanism by Denk and Botsaris, ${ }^{33,34}$ where at a supercooling of $3{ }^{\circ} \mathrm{C}$ and 350 RPM stirring in a stirred tank crystallizer, a crop of product crystals had $100 \%$ right handedness when seeded with a single right handed crystal. The equivalent outcome was reported for seeding with a levorotatory crystal. This would indicate that secondary nucleation took place due to either dendrite coarsening or collision breeding within the crystallizer, i.e. the entire product crystals were originated directly from the seed crystal. At higher supercoolings of greater than $7^{\circ} \mathrm{C}$, 
primary nucleation was observed. This was shown from a mixture of 50:50 left and right handed product crystals obtained, suggesting that if spontaneous nucleation occurred, a mixture of both left and right handed crystals would be the evidence. This outcome was also supported by Martin et al. ${ }^{35}$

The hypothesis investigated here is that a different nucleation mechanism could be observed in an OBC compared to that in a STC due to different styles of mixing, or mixing mechanisms, while all operational conditions remain constant, such as supersaturation, temperature, mixing intensity and so on. In an earlier work of seeded sodium chlorate crystallization at a supercooling of $1^{\circ} \mathrm{C}$, it was observed that in the STC, all of the product crystals bore the same handedness as the seed crystal, indicating that secondary nucleation directly from the seed was the source of product crystals. This will be referred to as "seed-similar" nucleation hereafter. For the OBC, on the other hand, the product crystals were never more than $96 \%$ similar to the seed crystal, suggesting an alternative nucleation mechanism that facilitates a "seed-dissimilar" nucleation model. The scraping action of the baffle outer edge against the inner surface of the crystallizer wall was proposed as the cause of generation of the incorrect enantiomorph in the OBC. To test this hypothesis, the scraping was removed from the $\mathrm{OBC}$, while it was introduced to the STC. The opposite results were obtained, i.e. the un-scraped OBC produced much more seed-similar crystals and the scraped STC produced seed-dissimilar crystals, which verified this hypothesis. ${ }^{36}$ The objective of this work is to investigate how the identified nucleation mechanism would vary with mixing intensity and local fluid flow mechanics.

\section{Experimental setup and procedure}

For this current work, two types of crystallizer were investigated, each had two possible configurations: with and without scraping. This allowed the nucleation mechanism to be further probed at various mixing intensities in both crystallizers, with the aims of reinforcing and consolidating these previous hypotheses and findings, as well as discovering new findings and extending the investigation through rigorous design of experiment.

\subsection{The stirred tank crystallizer}

Fig. 1 shows the setup of the STC. The STC consisted of a jacketed round bottom glass vessel with a working volume of $500 \mathrm{~mL}$. A curved, PTFE paddle type stirrer was driven by the overhead motor and was of diameter $80 \mathrm{~mm}$ and width $15 \mathrm{~mm}$. In order to introduce scraping, the impeller can be pressed tightly against the vessel bottom due to the curvature of the paddle. Raising the impeller allowed the scraping effect to be removed. Stainless steel wall baffles were introduced via a stainless steel flange. Four stainless steel baffles were spaced equally $90^{\circ}$ apart and extended approximately $30 \mathrm{~mm}$ into the fluid. This allowed the system to be well mixed, ${ }^{37}$ yet provided the stirrer a reasonable range of clearances from the vessel bottom without colliding with the

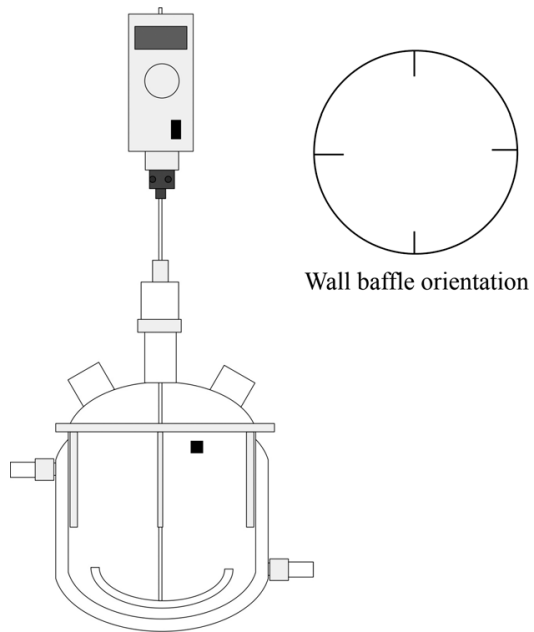

Fig. 1 Image of the STC setup (not to scale). Jacket connectors allowed temperature control via water bath. The steel wall baffles were suspended by the flange at the top of the vessel and orientated according to the schematic at the top right of the figure. The stirrer height was set by clamping the shaft at the overhead stirrer. The seed location is indicated by the filled square.

baffles. With a working volume of $500 \mathrm{~mL}$, the height of the liquid in the vessel was approximately $80 \mathrm{~mm}$. The rotation was adjustable from 50 to around 2000 RPM.

The energy dissipation of a stirred tank crystallizer is defined by: ${ }^{38}$

$$
\frac{P}{V}=\frac{P_{\mathrm{O}} \rho N_{\mathrm{s}}{ }^{3} D_{\mathrm{S}}{ }^{5}}{V_{\mathrm{L}}}
$$

where $P / V$ is the energy dissipation (power per unit volume) (W m $\left.{ }^{-3}\right), \rho$ the fluid density $\left(1456 \mathrm{~kg} \mathrm{~m}^{-3}\right.$ at $\left.40{ }^{\circ} \mathrm{C}^{39}\right), N_{\mathrm{s}}$ the speed of the stirrer (rps), $D_{\mathrm{S}}$ the diameter of the stirrer $(0.08 \mathrm{~m})$, and $V_{\mathrm{L}}$ the volume of liquid in the STC $\left(0.0005 \mathrm{~m}^{3}\right)$. $P_{\mathrm{O}}$ is the dimensionless power number of the agitator which was estimated at 1 based on data from Nagata. ${ }^{40}$

\subsection{The oscillatory baffled crystallizer}

Fig. 2 shows the setup of the OBC. The OBC consisted of a jacketed glass column of an internal diameter of $50 \mathrm{~mm}$ and a height of approximately $500 \mathrm{~mm}$. This gave a working volume of $500 \mathrm{~mL}$. The vessel was held in position by a stainless steel flange. Two sets of baffle strings were used in order to create scraped and non-scraped systems. The first baffle string comprised 4 PTFE baffles with an outer diameter of $50 \mathrm{~mm}$, an orifice diameter of $24 \mathrm{~mm}$ and a thickness of $3 \mathrm{~mm}$. The baffles were spaced at $65 \mathrm{~mm}$ by stainless steel spacers and tightly fit against the wall of the column, allowing the actions of scraping. The second baffle string consisted of 4 PTFE baffles with an outer diameter of $40 \mathrm{~mm}$, an orifice diameter of $17 \mathrm{~mm}$ and a thickness of $3 \mathrm{~mm}$. Again, the baffles were spaced at $65 \mathrm{~mm}$ with stainless steel spacers. In this case, there was a gap between the outer edges of the baffles and the inner surface of the column, leading to no scraping 


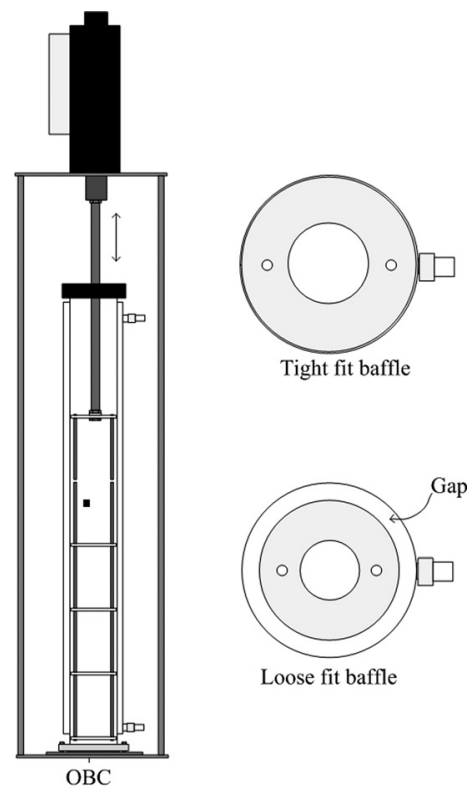

Fig. 2 Image of the $O B C$ setup (not to scale). The operating configuration of the OBC is shown to the left. A top view of the cross sectional area of the tight fitting baffle is shown top right and the loose fitting baffle shown bottom right. The seed location is indicated by the filled square.

against the vessel wall when oscillated by means of a linear motor (Copley Controls Corp.), which was held by an aluminium/plastic frame above the OBC. Frequencies of $0-10 \mathrm{~Hz}$ and amplitudes of $0-50 \mathrm{~mm}$ were possible. The selected trough to peak amplitude for the OBC was $30 \mathrm{~mm}$ (roughly half the baffle spacing and allowable by the hardware).

The energy dissipation of the OBC is estimated using the quasi steady flow model proposed by Baird and Stonestreet: ${ }^{41}$

$$
\frac{P}{V}=\frac{2 \rho N_{\mathrm{b}}}{3 \pi C_{\mathrm{D}}^{2}}\left(\frac{1-\alpha^{2}}{\alpha^{2}}\right) x_{\mathrm{o}}^{3}(2 \pi f)^{3}
$$

where $N_{\mathrm{b}}$ is the number of baffles per unit length of OBC (15.38 $\left.\mathrm{m}^{-1}\right), C_{\mathrm{D}}$ the discharge coefficient of the baffles (taken as 0.7$), x_{\mathrm{o}}$ the centre to peak amplitude of oscillation $(0.015 \mathrm{~m}), f$ the oscillation frequency $(\mathrm{Hz})$ and $\alpha$ the baffle free area ratio $(0.23$ for the tight fit baffles, 0.48 for the loose fit baffles). The free area ratio, $\alpha$ is defined by the area of the orifice divided by the tube cross-sectional area and also takes into account the gap around the baffle edge (if present).

Three energy dissipation values (low, medium and high) were achieved by changing the oscillation frequencies in the OBC and the stirrer speed in the STC, as given in Table 1. For the purpose of comparison, similar energy dissipation was used as the basis for each of the devices. ${ }^{26}$

\subsection{Experimental procedure}

As with the previous work, ${ }^{36}$ the crystallizers were temperature controlled by a Grant GP200R2 water bath and the temperature was monitored using stainless steel T-type thermocouples. The
Table 1 Mixing conditions for each crystallizer. The mixing speed is given to the left and the approximate energy dissipation $\left(\mathrm{W} \mathrm{m}^{-3}\right)$ given in brackets to the right

\begin{tabular}{lcll}
\hline Mixing condition & STC (RPM) & $\begin{array}{l}\text { OBC tight } \\
\text { baffles (Hz) }\end{array}$ & $\begin{array}{l}\text { OBC loose } \\
\text { baffles (Hz) }\end{array}$ \\
\hline Low & $65(12)$ & $0.4(9)$ & $0.8(13)$ \\
Medium & $160(180)$ & $1.0(145)$ & $2.0(216)$ \\
High & $260(776)$ & $\begin{array}{c}1.6(595) \\
2(1162)\end{array}$ & $3.0(732)$ \\
& & $2(72)$
\end{tabular}

general procedure involved preparing an aqueous solution of laboratory grade sodium chlorate (sourced from Fisher Scientific UK, 99+\% purity) saturated at $31{ }^{\circ} \mathrm{C}, c=1.05 \mathrm{~g} \mathrm{NaClO}_{3}$ per $\mathrm{g} \mathrm{H}_{2} \mathrm{O}$. The solution was made using distilled water to the working volume of the crystallizer, and cured at $40{ }^{\circ} \mathrm{C}$ for one hour before being filtered using an oven heated, Whatman glass fiber filter (grade GF/C nominal pore size $1.2 \mu \mathrm{m}$ ). Solutions were held again at $40{ }^{\circ} \mathrm{C}$ for a further 30 minutes before being cooled, with agitation, to $30{ }^{\circ} \mathrm{C}, c^{*}=1.03 \mathrm{~g} \mathrm{NaClO}_{3}$ per $\mathrm{g}$ $\mathrm{H}_{2} \mathrm{O}$, giving a calculated supersaturation, $S$, of 1.02. On reaching $30{ }^{\circ} \mathrm{C}$, a single seed crystal of known handedness was washed with cold distilled water and dried using filter paper before being suspended by means of a wire in the crystallizing solution for three minutes whilst maintaining agitation. The seed crystal was then removed from the solution and the agitation stopped to allow the product crystals to grow quiescently overnight at $30^{\circ} \mathrm{C}$ to form larger crystals. The product crystals were separated, dried and analysed by polarimetry. Both the left handed (levorotatory) and right handed (dextrorotatory) crystals were separated from an average of 3000 individual crystals for each run and the similarity to the seed crystals examined and calculated. Each experiment was repeated a further two times to establish the repeatability of the data. The apparatus was thoroughly cleaned between trials using household detergent at $60{ }^{\circ} \mathrm{C}$ followed by three rinses with hot water. The apparatus was allowed to dry prior to use.

\section{Results and discussion}

Before presenting the main results, a number of benchmark experiments were performed. In order to verify that no spontaneous nucleation could occur as a result of surface phenomena, the first set of experiments were conducted according to the procedure described earlier, except no seeds were used. Only the blank wire that was used for holding the single seed crystal was suspended in the solution. The crystallizers containing the supersaturated solutions were left overnight and it was found that no crystals were seen in any system (both scraped and un-scraped devices). The same outcome was observed three times in each device, indicating that the possibility of either the presence of a foreign body (the blank wire) or entraining foreign particles to initiate heterogeneous nucleation was not observable under the tested conditions. This suggests that for these experiments, the nucleation of product crystals stemmed entirely from the introduction of a single sodium chlorate seed crystal. 
The next set of benchmark tests were conducted in the previously described manner, but no mixing at all was applied to the crystallizers. This was to verify the hypothesis that it is the mixing mechanism that has an effect on the secondary nucleation. When no mixing was applied before, during and after the seeding, it was seen that all product crystals bore the same handedness as the seed crystal in both devices. Again, three repeats were done in both the OBC and STC systems. This outcome would suggest that in quiescent solutions, where the seed boundary layer has not been disturbed, the solute molecules would adhere to the seed in the bulk solution, thus providing seed-similar nucleation of products. This is in contrast to the findings of Denk and Botsaris, who found that the percentage similarity to the seed crystal was around $60 \%$ at $1{ }^{\circ} \mathrm{C}$ supercooling. ${ }^{33}$ The reason for the difference between their report and this current work may lie in the fact that while Denk and Botsaris were adding the wetted seed crystal to the solution, it "was rotated slightly to dissipate whatever water film might be adhering to its surfaces". ${ }^{33}$ The seed was added dry to the solution in the experiments reported here.

The final set of benchmarking experiments involved halting the agitation prior to seeding, rather than after seeding. This was to further verify the above hypothesis. As with the previous trials, it was found that all of the seeds were of the same handedness as the seed crystal. It would indicate that the mixing mechanism would be the only likely cause of deviation from $100 \%$ similarity to the seed either through affecting the seed boundary layer, or via the previously identified scraping action.

The results from the seeded crystallizations in the STC are presented in Table 2.

From the data of the un-scraped STC on the left of Table 2, it can be seen that the similarity to seed is consistently $100 \%$ for all power dissipations. While no mechanism was expected in the un-scraped STC to promote nucleation of the oppositeto-seed enantiomorph, increasing the power dissipated into the system would be expected to increase the rate of secondary nucleation by either crystal-crystal or crystal-crystallizer collisions, ${ }^{42,43}$ without influencing the product crystal handedness.

When scraping was introduced to the STC by simply having the impeller tightly pressed against the bottom of the vessel, seed-dissimilar nucleation did occur (the right side of Table 2). This outcome was expected from the previous work. ${ }^{36}$ It would seem reasonable to infer that as the mixing intensity increased in a system where a seed-dissimilar nucleation mechanism exists, one of two outcomes could be seen. The first possible scenario could be that the rate of seed-dissimilar nucleation because of the scraping mechanism would increase due to more scrapes per unit time (in this case rotations per second) resulting in a decrease in the percentage similarity to the seed of the crystals produced. The second possible outcome that may be observed could be the rate of seed-similar nucleation being enhanced due to either crystal-crystal or crystal-crystallizer collisions occurring more frequently because of the increased mixing. However the data in Table 2 suggest that neither scenario was observed. In fact, analysis of the data (Kruskal-Wallis ${ }^{44}$ ) suggests that there is no statistical significance in the differences between the results at various mixing intensities $(P=0.7326)$. These experiments suggest that although scraping did cause the formation of product crystals of the opposite enantiomorphism to the seed crystal, the energy dissipation at which scraping is applied had little or no influence on the rate of this seed-dissimilar nucleation. This could perhaps be due to both seed-similar and seed-dissimilar nucleation rates increasing with mixing intensity, resulting in no net change in the average similarity.

The experiments conducted in the STC were then repeated in the OBC. The results of these tests are given in Table 3.

Considering the scraped $\mathrm{OBC}$ first (the right side of Table 3), seed-dissimilar nucleation did occur, leading to the percentage of similarity to the seed being lower than $100 \%$. It appears, however, that there is no clear trend with the change in mixing intensity (frequency). Indeed, no statistical significance can be found in the difference between the outcomes under the three conditions $(P=0.1479)$. Similarly, with the un-scraped OBC (the left side of Table 3), some seed-dissimilar nucleation also took place. Analysis of the data of the un-scraped set up suggests again that there is no significant difference between each mixing intensity $(P=0.4298)$. In terms of trends found in both the scraped STC and scraped OBC, the effect of mixing (stirring vs. oscillation) had little impact. Statistical analysis of these data shows there is no significant difference in the percentage similarity between the scraped OBC and the scraped STC $(P=0.2004)$.

The seed-dissimilar mechanism took place in the un-scraped OBC, while it was not observed in the un-scraped STC. The statistical difference in the percentage similarity between the two un-scraped set ups is significant $(P=0.0005)$. Why is this? Previous work on the numerical modelling of flow patterns in both tight fit and loose fit baffle

Table 2 Percentage similarity to the seed crystal for the seeded crystallization in both the un-scraped (left) and scraped (right) STC

\begin{tabular}{|c|c|c|c|c|c|c|c|}
\hline Un-scraped sample & \multicolumn{3}{|c|}{ Mixing intensity } & Scraped sample & \multicolumn{3}{|c|}{ Mixing intensity } \\
\hline 1 & 100.00 & 100.00 & 100.00 & 1 & 92.62 & 100.00 & 93.62 \\
\hline 3 & 100.00 & 100.00 & 100.00 & 3 & 98.77 & 92.24 & 97.35 \\
\hline Average & 100.00 & 100.00 & 100.00 & Average & 95.33 & 97.09 & 95.77 \\
\hline
\end{tabular}


Table 3 Percentage similarity to the seed crystal for the seeded crystallization in both the un-scraped (left) and scraped (right) OBC at a fixed centre to peak amplitude of $15 \mathrm{~mm}$

\begin{tabular}{|c|c|c|c|c|c|c|c|}
\hline \multirow[t]{2}{*}{ Un-scraped sample } & \multicolumn{3}{|c|}{ Mixing intensity } & \multirow[t]{2}{*}{ Scraped sample } & \multicolumn{3}{|c|}{ Mixing intensity } \\
\hline & Low & Medium & High & & Low & Medium & High \\
\hline 1 & 100.00 & 98.86 & 99.58 & 1 & 94.02 & 99.00 & 93.09 \\
\hline 2 & 96.99 & 99.49 & 99.83 & 2 & 94.89 & 93.43 & 93.20 \\
\hline 3 & 99.88 & 98.69 & 99.01 & 3 & 96.31 & 92.90 & 90.49 \\
\hline Average & 98.96 & 99.01 & 99.47 & Average & 95.07 & 95.11 & 92.26 \\
\hline Standard error & 0.98 & 0.24 & 0.24 & Standard error & 0.67 & 1.95 & 0.89 \\
\hline
\end{tabular}

arrangements in the OBC showed that more shear was predicted in the latter device than in the former. ${ }^{45}$ The seed-dissimilar nucleation in the un-scraped OBC may be linked with the increased shear at the gap, even though it has previously been shown that the introduction of a gap in the OBC increased the mixing time, i.e. the larger the gap is, the poorer the mixing is. ${ }^{46}$ This would suggest that the local fluid flow condition in the un-scraped OBC could have played a deciding role on the nucleation mechanism.

To put this hypothesis to the test, lower oscillation amplitudes of 7 and $15 \mathrm{~mm}$ were used at a fixed frequency, so that the amount of fluid shearing at the gap is effectively reduced as the shear (strain) rate is proportional to the change of oscillatory velocity $\left(x_{\mathrm{o}} f\right)$ at the gap as

$$
\dot{\gamma} \propto \frac{x_{\mathrm{o}} f}{D}
$$

where $\dot{\gamma}$ is the fluid shear rate $\left(\mathrm{s}^{-1}\right)$ and $D$ is the baffle diameter (m). ${ }^{47}$

Clearly, a reduction of the amplitude at a fixed frequency reduces the value of the oscillatory velocity, $x_{\mathrm{o}} f$, and therefore the shear (strain) rate of the system. The results of the varied amplitude experiments in both the scraped and un-scraped OBC are presented in Table 4.

Examination of the data from the un-scraped OBC (the left side of Table 4) reveals that decreasing the amplitude, i.e. the shear (strain) effect, increased the similarity to the seed, and $100 \%$ similarity to the seed was achieved at the lowest amplitude of $7 \mathrm{~mm}$, where the shear effect was at its lowest. Increasing the amplitude gave a significant difference in the outcome of the experiment $(P=0.0349)$. The data continue to suggest that the local fluid mechanical conditions could influence nucleation mechanisms. To further confirm this, the same conditions were tried in the scraped OBC as shown the right side of Table 4 . A clear trend of the similarity to the seed increasing towards $100 \%$ with reducing oscillation amplitude is evident here, with a significant statistical difference $(P=0.0496)$. It seems that by reducing the effective scraped area via the length of the scraping in the system, the local shearing effect was effectively reduced, leading to the significant reduction of the amount of seed-dissimilar nucleation. The results suggest that both the overall mixing intensity and the local fluid mechanical conditions during the seeding within a crystallizer are equally important in influencing nucleation.

The question remains as how, exactly, the seed-dissimilar crystals were formed in the scraped and un-scraped systems. It could be speculated that the seed boundary layer could be the key factor in deciding whether or not nucleation would be seed-similar or seed-dissimilar. In the $\mathrm{OBC}$, the fluid flow regime could disrupt this boundary layer differently, giving different pre-nucleation conditions ${ }^{48}$ to those seen in the STC. For the scraped systems, the results presented here resemble an undergraduate chemistry lab project using a spatula scraping the surface of a beaker containing supersaturated solution, but the exact mechanism becomes harder to elucidate, perhaps with a combined scraping and fluid flow regime model in effect. As Davey et al. pointed out, determination of the crystal nucleus characteristics (such as size, shape, and, particularly, the enantiomorph) will require technology and data that are as yet unavailable. ${ }^{49}$

\section{Conclusions}

In conclusion, it was previously found that when a scraping mechanism is introduced to either a stirred tank or

Table 4 Percentage similarity to the seed crystal for the seeded crystallization in the scraped (left) and un-scraped (right) OBCs. These tests were conducted at a fixed frequency of $2 \mathrm{~Hz}$, the approximate energy dissipation $\left(\mathrm{W} \mathrm{m}^{-3}\right)$ is given in brackets next to the amplitude

\begin{tabular}{lccccrr}
\hline Un-scraped sample & \multicolumn{2}{l}{ Amplitude, $x_{\mathrm{o}}(\mathrm{mm})$} & \multicolumn{2}{c}{ Scraped sample } & \multicolumn{2}{c}{ Amplitude, $x_{\mathrm{o}}(\mathrm{mm})$} \\
\hline & $3.5(3)$ & $7.5(27)$ & $15(216)$ & & $3.5(14)$ & $7.5(145)$ \\
1 & & & & & $15(1162)$ \\
2 & 100.00 & 99.96 & 98.86 & 1 & 97.85 & 98.12 \\
3 & 100.00 & 99.94 & 99.49 & 2 & 100.00 & 95.72 \\
& 100.00 & 99.42 & 98.69 & 3 & 99.91 & 98.39 \\
Average & & & & & 93.12 \\
Standard error & 100.00 & 99.77 & 99.01 & Average & 99.25 & 97.41 \\
& 0.00 & 0.18 & 0.24 & Standard error & 0.70 & 0.85
\end{tabular}


oscillatory baffled crystallizer containing a supersaturated solution of sodium chlorate, nuclei of opposite enantiomorphism to the seed crystal are found in the crop of product crystals. ${ }^{36}$ This paper reported that no real change in the percentage of similarity has been seen in the two systems when the mixing intensity (in terms of frequency and stirring rate) was altered; further demonstrated that local fluid mechanical conditions (e.g. shearing effect due to the oscillatory amplitude) can also lead to the formation of seed-dissimilar crystals. By reducing such an effect, the un-scraped OBC gave $100 \%$ similarity to the seed at the lowest amplitude (shear effect). The results of this work indicate that the action of scraping generates nuclei of the opposite enantiomorphism to the seed, so does the local mixing/shearing conditions in a crystallizer.

\section{Acknowledgements}

The authors thank EPSRC and the EPSRC Centre for Innovative Manufacturing in Continuous Manufacturing and Crystallisation and Heriot-Watt University for the matching funding for this work. Thanks are expressed to: Chris Price and Alex Caillet of GSK; Gerry Steele and Amy Robertson of Astra Zeneca; Kevin Girard of Pfizer; Thomas Rammeloo of Johnson and Johnson; Jan Sefcik of The University of Strathclyde and the other Academics of the Continuous Crystallization Discussion Group (CCDG) for the most helpful monthly meetings. The authors thank Alison Nordon and Jaclyn Dunn of The University of Strathclyde for the most helpful discussion of statistical analysis.

\section{Notes and references}

1 S. W. Young, J. Am. Chem. Soc., 1911, 33, 148-162.

2 J. Ulrich and C. Strege, J. Cryst. Growth, 2002, 237-239, Part 3, 2130-2135.

3 J. W. Mullin and K. D. Raven, Nature, 1961, 190, 251-251.

4 J. W. Mullin and K. D. Raven, Nature, 1962, 195, 35-38.

5 J. Nývlt, J. Cryst. Growth, 1968, 3-4, 377-383.

6 J. Nývlt, R. Rychlý, J. Gottfried and J. Wurzelová, J. Cryst. Growth, 1970, 6, 151-162.

7 K. Liang, G. White, D. Wilkinson, L. J. Ford, K. J. Roberts and W. M. L. Wood, Ind. Eng. Chem. Res., 2004, 43, 1227-1234.

8 A. Myerson, Handbook of Industrial Crystallization, Butterworth Heinemann, 2002.

9 T. P. Melia and W. P. Moffitt, Ind. Eng. Chem. Fundam., 1964, 3, 313-317.

10 H. E. C. Powers, Ind. Chem., 1963, 39, 351.

11 T. P. Melia and W. P. Moffitt, J. Colloid Sci., 1964, 19, 433-447.

12 K. Y. Min and W. I. Goldburg, Phys. Rev. Lett., 1993, 71, 569.

13 J. Chen, B. Sarma, J. M. B. Evans and A. S. Myerson, Cryst. Growth Des., 2011, 11, 887-895.
14 T. Buhse, D. Durand, D. Kondepudi, J. Laudadio and S. Spilker, Phys. Rev. Lett., 2000, 84, 4405-4408.

15 W. C. Chen, D. D. Liu, W. Y. Ma, A. Y. Xie and J. Fang, J. Cryst. Growth, 2002, 236, 413-419.

16 D. Erdemir, A. Y. Lee and A. S. Myerson, Acc. Chem. Res., 2009, 42, 621-629.

17 E. L. Paul, H.-H. Tung and M. Midler, Powder Technol., 2005, 150, 133-143.

18 X. Ni, Innovations Pharm. Technol., 2006, 20, 90-96.

19 NiTech Solutions Ltd, http://www.nitechsolutions.co.uk, Accessed 18th March 2011.

20 X. Ni, H. Jian and A. Fitch, Trans. IChemE, 2003, 81, 842-853.

21 M. R. Hewgill, M. R. Mackley, A. B. Pandit and S. S. Panu, Chem. Eng. Sci., 1993, 48, 799-809.

22 M. R. Mackley, G. M. Tweedle and I. D. Wyatt, Chem. Eng. Sci., 1990, 45, 1237-1242.

23 S. Lawton, G. Steele, L. Shering, L. Zhao, I. Laird and X. Ni, Org. Process Res. Dev., 2009, 13, 1357-1363.

24 X. Ni, Y. S. De Gelicourt, M. H. I. Baird and N. V. Rama Rao, Can. J. Chem. Eng., 2001, 79, 444-448.

25 X. Ni and A. Liao, Chem. Eng. J., 2010, 156, 226-233.

26 C. M. Chew and R. I. Ristic, AIChE J., 2005, 51, 1576-1579.

27 NiTech Solutions Ltd., Clients' data, unpublished work.

28 C. W. Bunn, in Chemical Crystallography, Oxford Clarendon Press, Oxford, 1945, ch. 2, p. 39.

29 R. M. Pagni and R. N. Compton, Cryst. Growth Des., 2002, 2, 249-253.

30 C. Mayer and R. Lacmann, Chem. Eng. Technol., 1997, 20, 633-640.

31 D. K. Kondepudi, K. L. Bullock, J. A. Digits, J. K. Hall and J. M. Miller, J. Am. Chem. Soc., 1993, 115, 10211-10216.

32 D. K. Kondepudi, R. J. Kaufman and N. Singh, Science, 1990, 250, 975-976.

33 E. G. Denk and G. D. Botsaris, J. Cryst. Growth, 1972, 13-14, 493-499.

34 E. G. Denk and G. D. Botsaris, J. Cryst. Growth, 1972, 15, 57-60.

35 B. Martin, A. Tharrington and X. l. Wu, Phys. Rev. Lett., 1996, 77, 2826-2829.

36 C. J. Callahan and X.-W. Ni, Cryst. Growth Des., 2012, 12, 2525-2532.

37 N. A. Mitchell, P. J. Frawley and C. T. Ó'Ciardhá, J. Cryst. Growth, 2011, 321, 91-99.

38 R. K. Sinnott, in Coulson and Richardson's Chemical Engineering Volume 6 - Chemical Engineering Design, ed. R. K. Sinnott, Butterworth-Heinemann, Oxford, Third edn, 1999, ch. 10, pp. 471-473.

39 A. Seidell, in Solubilities of inorganic and metal organic compounds, Van Nostrand Company, New York, Third edn, 1940, vol. 1, p. 1250.

40 S. Nagata, Mixing: Principles and Applications, Kodansha Ltd, Tokyo, 1975.

41 M. H. I. Baird and P. Stonestreet, Trans. IChemE, 1995, 73, 503-511.

42 T. W. Evans, G. Margolis and A. F. Sarofim, AIChE J., 1974, 20, 950-958.

43 L. G. Bauer, R. W. Rousseau and W. L. McCabe, AIChE J., 1974, 20, 653-659. 
44 The MathWorks Inc., http://www.mathworks.co.uk/help/stats/ kruskalwallis.html, Accessed 11th July 2013.

45 M. E. Mackay, M. R. Mackley and Y. Wang, Trans. IChemE, 1991, 69, 506-513.

46 X. Ni and C. C. Stevenson, J. Chem. Technol. Biotechnol., 1999, 74, 587-593.
47 X. Ni, J. A. Cosgrove, A. D. Arnott, C. A. Greated and R. H. Cumming, Chem. Eng. Sci., 2000, 55, 3195-3208.

48 D. Gebauer and H. Colfen, Nano Today, 2011, 6, 564-584.

49 R. Davey, S. L. M. Schroeder and J. H. ter Horst, Angew. Chem., Int. Ed., 2013, 52, 2166-2179. 